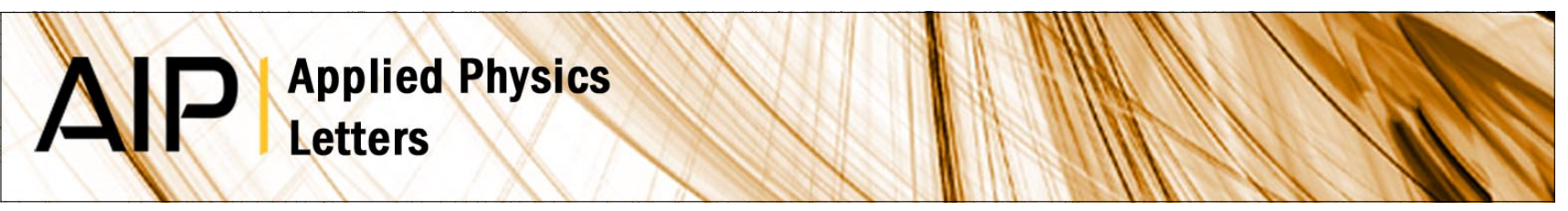

\title{
Direct imaging of the spatial diffusion of excitons in single semiconductor nanowires
}

M. A. Fickenscher, H. E. Jackson, L. M. Smith, J. M. Yarrison-Rice, J. H. Kang et al.

Citation: Appl. Phys. Lett. 99, 263110 (2011); doi: 10.1063/1.3671367

View online: http://dx.doi.org/10.1063/1.3671367

View Table of Contents: http://apl.aip.org/resource/1/APPLAB/v99/i26

Published by the American Institute of Physics.

\section{Related Articles}

Tantalum nitride superconducting single-photon detectors with low cut-off energy

Appl. Phys. Lett. 100, 062601 (2012)

An empirical law for the band gaps of $\mathrm{MgZnO}$ nanowires

J. Appl. Phys. 110, 124315 (2011)

Single-particle and collective excitations in quantum wires made up of vertically stacked quantum dots: Zero magnetic field

J. Chem. Phys. 135, 124704 (2011)

Intrinsic and external strains modulated electronic properties of $\mathrm{GaN} / \mathrm{InN}$ core/shell nanowires

J. Appl. Phys. 110, 054308 (2011)

Threshold conductivity switching in sulfurized antimony selenide nanowires

Appl. Phys. Lett. 99, 103101 (2011)

\section{Additional information on Appl. Phys. Lett.}

Journal Homepage: http://apl.aip.org/

Journal Information: http://apl.aip.org/about/about_the_journal

Top downloads: http://apl.aip.org/features/most_downloaded

Information for Authors: http://apl.aip.org/authors

\section{ADVERTISEMENT}

NEW!

iPeerReview

AlP's Newest App

$\frac{\Delta I P}{L}$
Authors...

Reviewers...

Check the status of

submitted papers remotely!

AIP Publishing 


\title{
Direct imaging of the spatial diffusion of excitons in single semiconductor nanowires
}

\author{
M. A. Fickenscher, ${ }^{1}$ H. E. Jackson, ${ }^{1}$ L. M. Smith, ${ }^{1, a)}$ J. M. Yarrison-Rice, ${ }^{2}$ J. H. Kang, ${ }^{3}$ \\ S. Paiman, ${ }^{3}$ Q. Gao, ${ }^{3}$ H. H. Tan, ${ }^{3}$ and C. Jagadish ${ }^{3}$ \\ ${ }^{1}$ Department of Physics, University of Cincinnati, Cincinnati, Ohio 45221, USA \\ ${ }^{2}$ Department of Physics, Miami University, Oxford, Ohio 45056, USA \\ ${ }^{3}$ Department of Electronic Materials Engineering, Research School of Physics and Engineering, \\ The Australian National University, Canberra, ACT 0200, Australia
}

(Received 14 August 2011; accepted 15 November 2011; published online 29 December 2011)

\begin{abstract}
We use spatially and temporally resolved photoluminescence to measure exciton diffusion in single zinc blende GaAs/AlGaAs core/shell and mixed phase InP nanowires. Excitons in the single phase GaAs/AlGaAs nanowires are seen to diffuse rapidly throughout the nanowire with a measured diffusion constant ranging from 45 to $100 \mathrm{~cm}^{2} / \mathrm{s}$, while in the mixed phase, InP nanowire electrons and holes are seen to rapidly localize to the quantum confined states in the zinc blende and wurtzite segments, respectively. The diffusion constant in the GaAs/AlGaAs nanowire is similar to the best hole mobilities observed in modulation doped heterostructures. (C) 2011 American Institute of Physics. [doi:10.1063/1.3671367]
\end{abstract}

Semiconductor nanowires (NWs) are increasingly being used in both traditional and novel configurations for a wide range of optoelectronic applications. The understanding of transport in these nanowires is important both for assessing the intrinsic material quality as well as the suitability for particular applications. Because of the quasi-one-dimensional geometry, Hall measurements are not accessible for characterization of nanowires, and so other techniques must be used to determine what factors influence their mobility. Electrical transport measurements in nanowires have utilized both field effect transistors as well as Schottky contacts to determine the mobility in nanowires, but these depend sensitively on the models used to interpret the data. ${ }^{1-5}$ In particular, the interaction of carriers with the lithographically defined contacts can be quite complex, and so determination of the mobility is necessarily indirect. Static optical imaging experiments in nanowires using cathodoluminescence and photocurrent imaging in a Schottky-Ohmic nanowire device have both been used to estimate the mobility in nanowires. ${ }^{6,7}$ In this letter, we report a technique that uses a pulsed laser to excite the excitons at a point on the nanowire and a high index solid immersion lens (SIL) to image the photoluminescence (PL) emitted from the expanding cloud of excitons with high spatial and temporal resolution. A similar technique has been used previously by a number of groups to measure the diffusion of exciton in $2 \mathrm{D}$ and $3 \mathrm{D}$ materials but not within a single nanostructure. ${ }^{8-12}$ As contrasting examples, we present measurements of exciton diffusion within a single zinc blende (ZB) GaAs/AlGaAs core-shell nanowire and in a single mixed phase zinc blende-wurtzite (ZB/WZ) InP nanowire. ${ }^{13}$ We measure a diffusion constant of 100 $\mathrm{cm}^{2} / \mathrm{s}$ for excitons at low temperatures $(10 \mathrm{~K})$ in single GaAs/AlGaAs nanowires of length $\sim 4 \mu \mathrm{m}$ which is equivalent through the Einstein relation to effective hole mobilities that approach $116000 \mathrm{~cm}^{2} / \mathrm{Vs}$, and within the mixed-phase

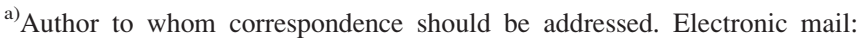
leigh.smith@uc.edu.
}

InP nanowire, we observe the rapid localization of excitons. In the following, we briefly discuss the nanowire growth and the experimental set up. We then present a theoretical interpretation of such experiments, the data, and finally our conclusions.

The GaAs/AlGaAs core-shell nanowires studied here were grown using the vapor liquid solid growth technique by metal organic chemical vapor deposition (MOCVD) with a $\mathrm{V} / \mathrm{III}$ ratio of 46.3 . The GaAs core growth was grown using a two temperature growth technique, which has been shown to result in a twin free single crystal nanowire. ${ }^{14-16}$ The core growth was initiated at an initial temperature of $450{ }^{\circ} \mathrm{C}$ for $1 \mathrm{~min}$, followed by the remainder of the core growth over $30 \mathrm{~min}$ at a lower temperature of $375^{\circ} \mathrm{C}$. The AlGaAs shell was then grown at $650{ }^{\circ} \mathrm{C}$ for 20 min with an $\mathrm{Al}$ vapor concentration of $26 \%$. A final $5 \mathrm{~nm}$ GaAs capping shell was added to prevent oxidation of the AlGaAs. Previous photoluminescence work on this nanowire series shows free exciton emission at $1.51 \mathrm{eV}$ with high quantum efficiency and a relatively long recombination lifetime of $600 \mathrm{ps}^{15-17}$ Thus, we expect that excitons in this sample to have a relatively long diffusion length.

The mixed-phase (ZB/WZ) InP nanowire studied was also grown by MOCVD at a V/III ratio of 700 using a growth temperature of $400{ }^{\circ} \mathrm{C}$ for $20 \mathrm{~min}$. Extensive characterization of this sample showed that it is a mixture of two crystal phases, predominantly $(80 \%)$ wurtzite with (20\%) zincblende inclusions. In such a structure, the holes are localized to the WZ sections while the electrons are localized to the ZB sections. ${ }^{18-20}$ For such a mixed phase crystal structure, we expect the exciton to exhibit a relatively short diffusion length as they thermalize to the lattice temperature and then rapidly become localized as the electrons and holes become captured into the lower energy confined states. ${ }^{18}$ To measure the spatial transport of excitons optically, we focus the excitation laser to a tightly focused spot as well as image the PL from the nanowire with very high spatial resolution onto a spectrometer slit. To achieve both these aims, we first 
disperse the nanowires onto the center of a hemispherical SIL. The SIL is a hemisphere of $4 \mathrm{~mm}$ glass (Ohara SLAH79) which has a high index of refraction, $n=2$. When the NWs are imaged using the SIL with a $50 \times / 0.5 \mathrm{NA}$ objective, the effective NA is increased by the index of refraction or a factor of $2 .^{21,22}$ The benefits of using a SIL are twofold: first, the $740 \mathrm{~nm}$ laser is more tightly focused, with the spot size decreased by a factor of $\mathrm{n}$ to $600 \mathrm{~nm}$ and second, the spatial resolution of the image of the $820 \mathrm{~nm}$ PL emitted from the nanowire is also increased by a factor of $n$ to $600 \mathrm{~nm}$. The SIL with the nanowires is then mounted onto the cold finger of a continuous flow helium cryostat operated at $10 \mathrm{~K}$. A circularly polarized $740 \mathrm{~nm} 76 \mathrm{MHz}$ pulsed titanium-sapphire laser is split into two paths, with part of the laser directed onto the sample and part detected by a fast photodiode which generates a start reference pulse for a time-to-amplitude converter (TAC) to enable the timeresolved PL measurements using time-correlated single photon counting. The system response is $50 \mathrm{ps}$.

To acquire the spatially resolved time scans, the laser spot is kept fixed at the center of the NW while a $200 \times$ magnified image of the NW is projected perpendicular to the entrance slit of the spectrometer (see Fig. 1). The specularly reflected laser light is removed with a sharp $750 \mathrm{~nm}$ Raman edge filter. Thus, a $4 \mu \mathrm{m}$ long nanowire becomes a $800 \mu \mathrm{m}$ long image across the slit. By moving a final lens, we scan this image of the nanowire across the $150 \mu \mathrm{m}$ entrance slit so that PL emitted from a $750 \mathrm{~nm}$ section of the wire can be detected by the fast phototube as a function of distance away from the laser spot fixed at the center of the nanowire. By taking time decays as a function of position, $\mathrm{x}$, away from the laser excitation spot, we can directly image the spatial expansion of excitons as a function of time after the laser excitation pulse and so obtain the exciton diffusion constant. The spectrometer was set for the peak PL emission energy $(1.51 \mathrm{eV}$ for $\mathrm{GaAs}$ and $1.50 \mathrm{eV}$ for $\mathrm{ZB} / \mathrm{WZ} \mathrm{InP})$ with an energy width of $6 \mathrm{meV}$ determined by the exit slit width.

Theoretically, the diffusion of excitons in the nanowire after photoexcitation by a laser pulse can be modeled by the one dimensional diffusion equation

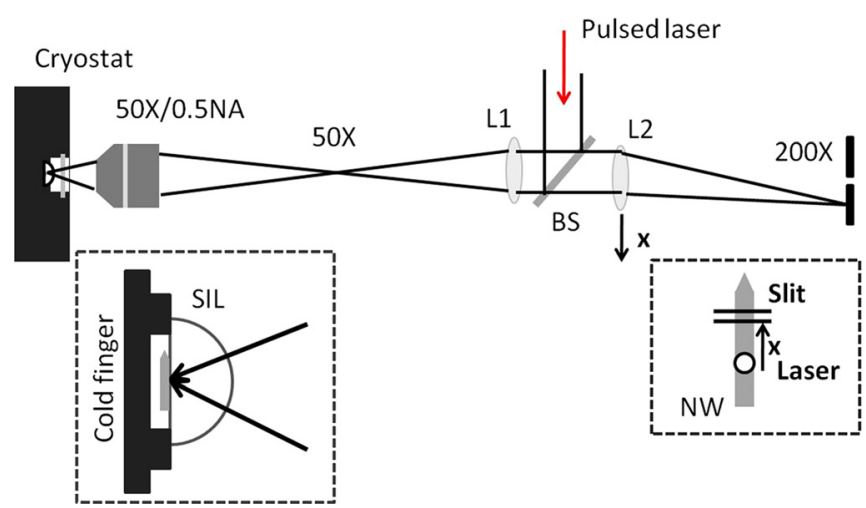

FIG. 1. (Color online) Schematic of experimental set-up. Pulsed $740 \mathrm{~nm}$ laser is focused onto a nanowire on the bottom of a SIL mounted on copper cold finger in a cryostat using a beam splitter and $50 \times / 0.5$ NA long working distance objective. Photoluminescence from the NW is imaged onto entrance slit of the spectrometer using lenses L1 and L2. Lens L2 is translated to position NW onto entrance slit of the spectrometer. Insets show detail of SIL and NW on cold finger and relative position of the laser spot to the entrance slit on the NW image.

$$
\frac{\partial \mathrm{n}}{\partial \mathrm{t}}=\mathrm{D} \frac{\partial^{2} \mathrm{n}}{\partial \mathrm{x}^{2}}-\frac{\mathrm{n}}{\tau_{1}}
$$

where $n(x, t)$ is the density of excitons as a function of position and time, $\mathrm{D}$ is the diffusion coefficient, and $\tau_{1}$ is the recombination lifetime of the exciton. The solution to the diffusion equation yields the spatial distribution of excitons as a function of position and time

$$
n(x, t) \propto \frac{1}{(4 \pi D t)^{\frac{3}{2}}} \exp \left[-\frac{x^{2}}{4 D t}\right] \exp \left[-\frac{t}{\tau_{1}}\right] .
$$

This solution consists of a Gaussian term, which expresses the expansion of the excitons away from the excitation point with time, and an exponential decay which models the decay of excitons with time through recombination. By taking spatial PL images in time (snapshots) after the laser pulse, the expanding Gaussians can be measured directly. One can show that the square of the FWHM $(\Delta)$ of the PL spatial distribution expands linearly with time as $\Delta^{2}=2.77(4 \mathrm{Dt}) .{ }^{8-12}$ The diffusion constant measured using this method is independent of the recombination lifetime of the excitons. As we will demonstrate, such measurements are a direct method of extracting the diffusion constant for the excitons in the nanowire. Because the hole is significantly more massive than the electron, the exciton diffusion constant will be determined by the hole diffusion constant, which is directly proportional to the hole mobility through the Einstein relation: $\mathrm{D}=\mu \mathrm{k}_{\mathrm{B}} \mathrm{T}$.

We present results first for the GaAs/AlGaAs nanowire and then for the mixed phase InP nanowire. The time decays are assembled into a 2D map of PL intensity where each point is the intensity of the PL at one instant in time and one particular spatial position with respect to the excitation laser pulse. A typical spatial-temporal map of the PL from a single GaAs/AlGaAs nanowire is shown in Fig. 2(a). To visualize the expansion more easily, the peak intensity at each time is normalized to unity. The spatial map shows that the spatial distribution is quite narrow at the earliest times and expands rapidly within the first 160 ps. After 160 ps, the spatial distribution is stationary suggesting that the exciton cloud has reached the end of the nanowire. In addition, one can also see that although the laser is focused at the center of the nanowire, the expansion is quite asymmetric with the exciton cloud apparently stopping in one direction while continuing to expand along the other. This may reflect the presence of stacking fault defects near the gold catalyst which were seen occasionally in TEM micrographs of wires from the same growth.

The time-resolved PL spatial map for the mixed-phase ZB/WZ InP nanowire displays a completely different behavior. While some small expansion is seen at the earliest times, the spatial distributions become completely stationary within 60 ps and do not expand at later times, even though the excitons are well away from the ends of the $8 \mu \mathrm{m}$-long nanowire. This localization is expected in this case since the electrons and holes are captured quite rapidly, within $50 \mathrm{ps}$, to the ZB and $\mathrm{WZ}$ sections (respectively) of the nanowire. ${ }^{18}$ As more fully described in the supplemental information, the $1.50 \mathrm{eV}$ emission energy corresponds to either the holes or the 

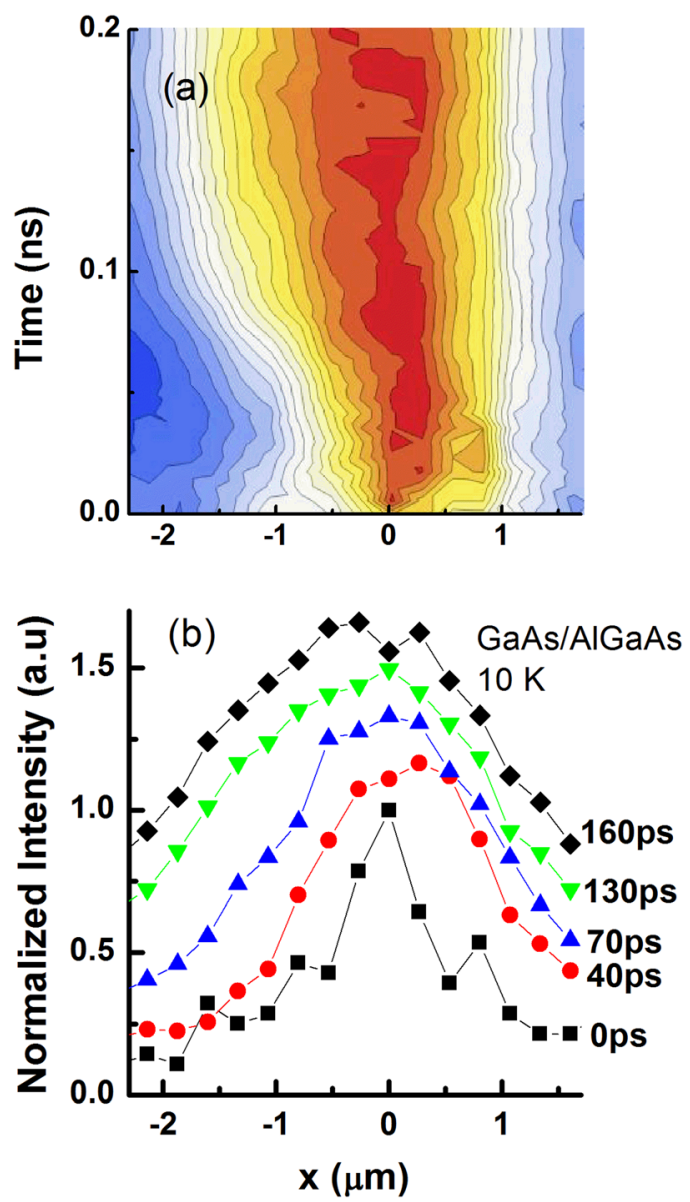

FIG. 2. (Color online) (a) Time-resolved spatially resolved PL false-color image from the GaAs/AlGaAs core-shell NW where maximum intensity is normalized to unity. Pulsed laser excitation spot is at $\mathrm{x}=0, \mathrm{t}=0$, and excitons are seen to diffuse rapidly. (b) Spatial profiles of exciton PL emitted from the nanowire at several times from 0 ps to 160 ps after the laser pulse excitation.

electrons strongly localized to the WZ or ZB segments, respectively. ${ }^{13}$ These quantum confined states are not mobile.

In order to calculate a diffusion constant, we extract the spatial distribution of the excitons from the time-resolved spatial scans as a function of time after the laser pulse. These spatial scans are shown in Figs. 2(b) and 3(b) below the maps. The FWHM of each spatial distribution is determined by fitting the scan at each time to a Gaussian. Because the spatial scans of the InP (Fig. 3(b)) are symmetric, this is easily done and the FWHM ${ }^{2}$ is displayed as squares in Fig. 4. Because the spatial expansion of excitons in the GaAs/ $\mathrm{AlGaAs}$ nanowires is asymmetric, the FWHM of the Gaussian distribution is determined in two ways. The circles in Fig. 4 display the resulting FWHM $^{2}$ as a function of time determined by fitting the entire distribution as a single Gaussian. The triangles show the $\mathrm{FWHM}^{2}$ for the distributions by doubling the half-width at half maximum (HWHM) measured from the expanding side of the distribution only and squaring. The solid lines show linear fits to this data during the rapid expansion of the excitons. As noted previously, for diffusion, the $\mathrm{FWHM}^{2}$ should expand with time as $\Delta^{2}=2.77(4 \mathrm{D}) \mathrm{t}$, where $\mathrm{D}$ is the diffusion constant. Thus, the diffusion constants are determined by taking the slope of the
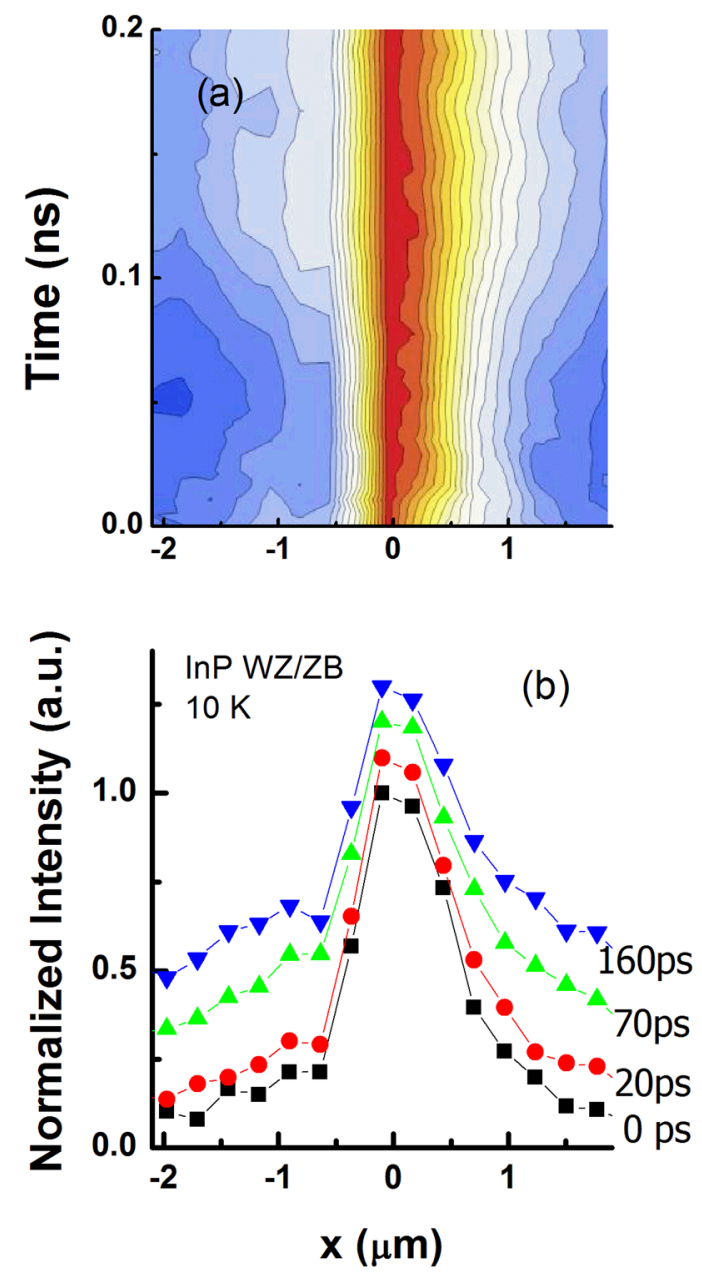

FIG. 3. (Color online) (a) Time-resolved spatially resolved PL false-color image from the mixed phase WZ/ZB InP NW where maximum intensity is normalized to unity. Pulsed laser excitation spot is at $\mathrm{x}=0, \mathrm{t}=0$, and excitons are seen to diffuse rapidly. (b) Spatial profiles of exciton PL emitted from the nanowire at several times from 0 ps to 160 ps after the laser pulse excitation.

line and dividing by 11.08 . For the $\mathrm{ZB} / \mathrm{WZ}$ InP nanowire, we determine a diffusion constant during the brief expansion phase of $25 \mathrm{~cm}^{2} / \mathrm{s}$. This corresponds through the Einstein relation to an effective hole mobility of $29000 \mathrm{~cm}^{2} / \mathrm{Vs}$ which is an order of magnitude higher than the highest mobility measured at $80 \mathrm{~K}$ for a doped $\mathrm{ZB}$ InP epilayer; ${ }^{23}$ this is perhaps not surprising since ionized impurity scattering is not present for excitons and the temperature is an order of magnitude lower here. The GaAs/AlGaAs nanowire diffusion constants range from a low of $45 \mathrm{~cm}^{2} / \mathrm{s}$ assuming expansion on both sides, to $100 \mathrm{~cm}^{2} / \mathrm{s}$ assuming expansion on one side. This corresponds to mobilities which range from 52000 $\mathrm{cm}^{2} / \mathrm{Vs}$ to $116000 \mathrm{~cm}^{2} / \mathrm{Vs}$. The higher estimate is as good as the best obtained for a p-type modulation-doped GaAs/ AlGaAs quantum well at similar temperatures. ${ }^{24,25}$ In both cases, the role of ionized impurity scattering and scattering from phonons is minimized.

In conclusion, we have used spatially and temporally resolved PL measurements to directly obtain the diffusion of excitons in single semiconductor nanowires. We find that the diffusion of excitons in two contrasting types of nanowires, GaAs/AlGaAs core/shell nanowire vs. InP mixed phase 


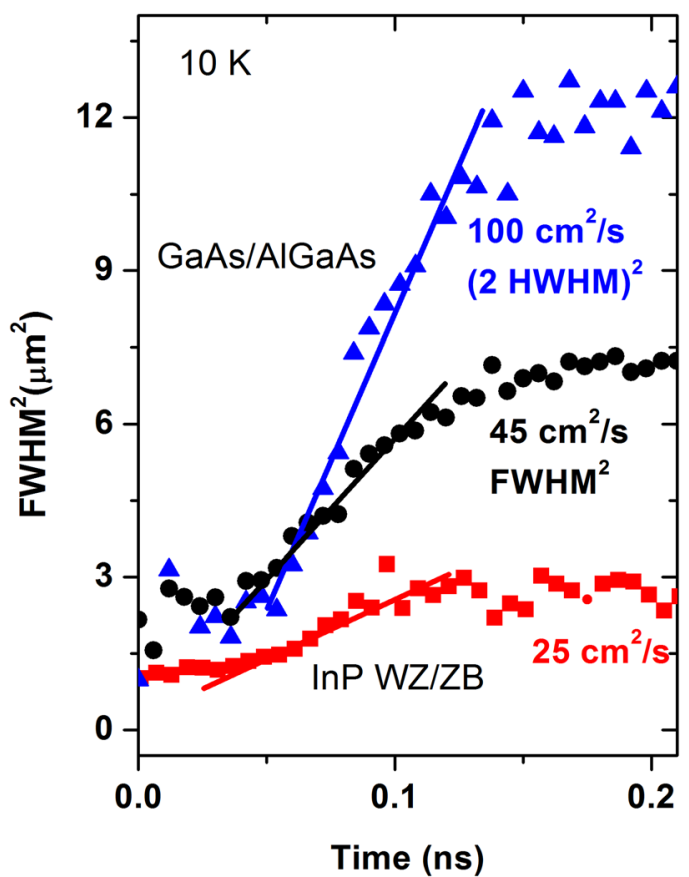

FIG. 4. (Color online) Square of the spatial widths $\left(\mathrm{FWHM}^{2}\right)$ of the emitted PL from the GaAs/AlGaAs core-shell NW and WZ/ZB InP nanowire as a function of time after the laser pulse excitation. Squares show exciton diffusion in WZ/ZB InP nanowires, while circles and triangles show $\mathrm{FWHM}^{2}$ and $(2 \mathrm{HWHM})^{2}$ distributions to account for asymmetric exciton expansion. Lines show fits to the expansion and the diffusion constants calculated from the slopes.

(WZ/ZB) nanowires are significantly different. For the high quality GaAs/AlGaAs NW, exciton diffusion is apparent from the central laser excitation spot outwards reaching to the ends of the nanowire. A measured diffusion constant of between $45 \mathrm{~cm}^{2} / \mathrm{s}$ and $100 \mathrm{~cm}^{2} / \mathrm{s}$ is found corresponding to effective mobilities through the Einstein relation between 52000 and $116000 \mathrm{~cm}^{2} / \mathrm{Vs}$, comparable to very high quality modulation doped quantum wells. In contrast, for the mixedphase $\mathrm{ZB} / \mathrm{WZ}$ InP nanowire, exciton diffusion is seen only during the time before the electrons and holes become trapped to the quantum confined states in the $\mathrm{ZB}$ and $\mathrm{WZ}$ sections. During the brief period while the excitons are diffusing, the diffusion constant is measured to be $22 \mathrm{~cm}^{2} / \mathrm{s}$ with a resulting hole mobility of $29000 \mathrm{~cm}^{2} /$ Vs. These results were obtained without the complication of electrical contacts by using an all optical technique for measuring the diffusive transport of excitons which provides a direct measure of the effective hole mobility of single semiconductor nanowires.
We acknowledge the financial support of the National Science Foundation through grants DMR-0806700, 0806572, 1105362, 1105121, and ECCS-1100489, and the Australian Research Council. The Australian National Fabrication Facility is acknowledged for access to the growth facilities used in this research.

${ }^{1}$ Z. Zhang, C. Jin, X. Liang, Q. Chen, and L. Peng, Appl. Phys. Lett. 88, 073102 (2006).

${ }^{2}$ Z. Y. Zhang, K. Yao, Y. Liu, C. H. Jin, X. L. Liang, Q. Chen, and L. M. Peng, Adv. Funct. Mater. 17, 2478 (2007).

${ }^{3}$ S. A. Dayeh, Semicond. Sci. Technol. 25, 024004 (2010).

${ }^{4}$ A. M. Katzenmeyer, F. Leonard, A. A. Talin, M. E. Toimil-Molares, J. G. Cederberg, J. Y. Huang, and J. L. Lensch-Falk, IEEE Trans. Nanotechnol. 10, 92 (2011).

${ }^{5}$ J. W. W. van Tilburg, R. E. Algra, W. G. G. Immink, M. Verheijen, E. P. A. M. Bakkers, and L. P. Kouwenhoven, Semicond. Sci. Technol. 25, 024011 (2010).

${ }^{6}$ A. Soudi, P. Dhakal, and Y. Gu, Appl. Phys. Lett. 96, 253115 (2010).

${ }^{7}$ L. Baird, C. P. Ong, R. A. Cole, N. M. Haegel, A. A. Talin, Q. Li, and G.

T. Wang, Appl. Phys. Lett. 98, 132104 (2011).

${ }^{8}$ L. M. Smith, D. J. Wolford, J. Martinsen, R. Venkatasubramanian, and S. K. Ghandhi, J. Vac. Sci. Technol. B 8, 787 (1990).

${ }^{9}$ L. M. Smith and J. P. Wolfe, Phys. Rev. B 51, 7521 (1995).

${ }^{10}$ G. D. Gilliland, A. Antonelli, D. J. Wolford, K. K. Bajaj, J. Klem, and J. A. Bradley, Phys. Rev. Lett. 71, 3717 (1993).

${ }^{11}$ J. C. Kim, D. R. Wake, and J. P. Wolfe, Phys. Rev. B 50, 15099 (1994).

${ }^{12}$ Z. Voros, R. Balili, D. Snoke, L. Pfeiffer, and K. West, Phys. Rev. Lett. 94, 226401 (2005).

${ }^{13}$ See supplemental material at http://dx.doi.org/10.1063/1.3671367 for a more detailed description and representative PL from samples.

${ }^{14}$ H. J. Joyce, Q. Gao, H. H. Tan, C. Jagadish, Y. Kim, X. Zhang, Y. N. Guo, and J. Zou, Nano Lett. 7, 921 (2007).

${ }^{15}$ J. H. Kang, Q. Gao, H. J. Joyce, H. H. Tan, C. Jagadish, Y. Kim, D. Y. Choi, Y. Guo, H. Xu, J. Zou et al. Nanotechnology 21, 035604 (2010).

${ }^{16}$ J. Kang, Q. Gao, H. J. Joyce, H. H. Tan, C. Jagadish, Y. Kim, Y. Guo, H. Xu, J. Zou, M. A. Fickenscher et al. Cryst. Growth Des. 11, 3109 (2011).

${ }^{17}$ S. Perera, M. A. Fickenscher, H. E. Jackson, L. M. Smith, J. YarrisonRice, H. J. Joyce, Q. Gao, H. H. Tan, C. Jagadish, X. Zhang et al., Appl. Phys. Lett. 93, 053110 (2008).

${ }^{18}$ K. Pemasiri, M. Montazeri, R. Gass, L. M. Smith, H. E. Jackson, J. Yarrison-Rice, S. Paiman, Q. Gao, H. H. Tan, C. Jagadish et al., Nano Lett. 9, 648 (2009).

${ }^{19}$ S. Paiman, Q. Gao, H. H. Tan, C. Jagadish, K. Pemasiri, M. Montazeri, H. E. Jackson, L. M. Smith, J. M. Yarrison-Rice, X. Zhang et al., Nanotechnology 20, 225606 (2009).

${ }^{20}$ J. Bao, D. C. Bell, F. Capasso, J. B. Wagner, T. Mårtensson, J. Trägårdh, and L. Samuelson, Nano Lett. 8, 836 (2008).

${ }^{21}$ S. M. Mansfield and G. S. Kino, Appl. Phys. Lett. 57, 2615 (1990).

${ }^{22}$ K. P. Hewaparakrama, A. Wilson, S. Mackowski, H. E. Jackson, L. M. Smith, G. Karczewski, and J. Kossut, Appl. Phys. Lett. 85, 5463 (2004).

${ }^{23}$ M. B. Kohanyuk, G. L. Lyakhu, I. P. Molodyan, and E. V. Russu, Indium Phosphide in Semiconductor Electronics, edited by S. I. Radaucan (Shtinca, Kishinev, 1988), p. 200.

${ }^{24}$ H. L. Stormer, A. C. Gossard, W. Wiegmann, R. Blondel, and K. Baldwin, Appl. Phys. Lett. 44, 139 (1984).

${ }^{25}$ W. I. Wang, E. E. Mendez, and F. Stern, Appl. Phys. Lett. 45, 639 (1984). 